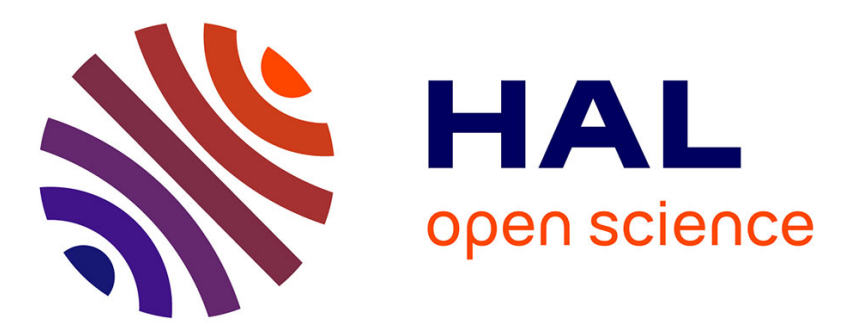

\title{
Hydrostatic pressure effect on the non-linear mechanics of filled rubbers: experiments and physico-mechanical approach
}

Jonathan Champagne, C Ovalle, S Cantournet, F. Lequeux, H. Montes, K Le-Gorju

\section{To cite this version:}

Jonathan Champagne, C Ovalle, S Cantournet, F. Lequeux, H. Montes, et al.. Hydrostatic pressure effect on the non-linear mechanics of filled rubbers: experiments and physico-mechanical approach. European Conference on Constitutive Models for Rubbers, Jun 2019, Nantes, France. pp.194, 10.1201/9780429324710-35 . hal-02174558

\section{HAL Id: hal-02174558}

https://hal-mines-paristech.archives-ouvertes.fr/hal-02174558

Submitted on 5 Jul 2019

HAL is a multi-disciplinary open access archive for the deposit and dissemination of scientific research documents, whether they are published or not. The documents may come from teaching and research institutions in France or abroad, or from public or private research centers.
L'archive ouverte pluridisciplinaire HAL, est destinée au dépôt et à la diffusion de documents scientifiques de niveau recherche, publiés ou non, émanant des établissements d'enseignement et de recherche français ou étrangers, des laboratoires publics ou privés. 


\title{
Hydrostatic pressure effect on the non-linear mechanics of filled rubbers: experiments and physico-mechanical approach
}

\author{
J. Champagne, C. Ovalle \& S. Cantournet \\ PSL Research University, Mines ParisTech, Centre des Matériaux, CNRS 7633 BP 87, F-91003 Evry Cedex, France
}

F. Lequeux \& H. Montes

PSL Research University, ESPCI Paris, Sciences et Ingénierie de la Matière Molle, Paris Cedex 5, France

K. Le-Gorju

Hutchinson SA, Research and Innovation Centre, Châlette-sur-Loing, France

\begin{abstract}
It has been known for several decades that rubbers exhibit a hydrostatic pressure-temperature superposition, interpreted by the free-volume theory. Moreover, our experimental results, on a filled rubber, demonstrate a hydrostatic pressure-temperature superposition which is two orders of magnitude larger than the one observed in pure rubbers. A new physico-mechanical model is proposed to account for the hydrostatic pressure effect responsible for a local glass transition temperature shift. It is suggested that it comes from the change of distances between aggregates. The predicted shift, given by the model, is found to be consistent with experimental data.
\end{abstract}

\section{INTRODUCTION}

The addition of "active" fillers -carbon black or silicain pure rubbers leads to a strong enhancement in mechanical properties (Bills \& al. 1960). Reinforcement goes beyond the mere increase in stiffness, showing improvements in the elongation at break, hysteresis and abrasion resistance. Hence, filled rubbers are of prior importance for many industrial applications. In some specific applications, the material is confined between stiffer parts resulting in hydrostatic pressure, generally excluded in modelling.

The hydrostatic pressure effect on mechanical properties has been widely investigated in pure rubbers for the last 70 years. Measurements of stress relaxation modulus (Fillers \& Tschoegl 1977), Young's modulus (Paterson 1964) or even dielectric properties (Cheng \& al. 1999) under superposed hydrostatic pressure and temperature were performed using high pressure chambers. More recently, hydrostatic pressure effect was investigated through shearcompression tests (Alkhader \& al. 2012). Unanimously, experimental results show that glass transition temperature rises with hydrostatic pressure by amounts varying from 0.15 to $0.3 \mathrm{~K}_{\mathrm{MPa}} \mathrm{MP}^{-1}$ depending on the material. These values are consistent with theoretical predictions based on the free-volume theory (Ferry \& Stratton 1960). This effect will be referred as "matrix pressure effect" in the following. To our knowledge, hydrostatic pressure effect has never been studied on filled rubbers.

In this paper, hydrostatic pressure effect on the thermo-mechanical behaviour of filled rubbers is shown to be two orders of magnitude greater than the one observed in pure rubbers. Observations are described under a physical framework allowing to develop a new physico-mechanical model accounting for local glass transition temperature.

\section{MATERIAL AND TEST SAMPLE}

The studied material is a poly(dimethylsiloxaneco-diphenylsiloxane) rubber highly filled with silica nanoparticles. Based on Nuclear Magnetic Resonance (NMR) measurements, the molar proportion of diphenylsiloxane groups is estimated at 5\%. Classical Thermo-Gravimetric Analysis (TGA) does not allow to scale the reinforcement due to a ceramic production from polymer during thermal degradation. However, the mass proportion of fillers is estimated at 30\%. From Differential Scanning Calorimetry (DSC) and Dynamic Thermo-Mechanical Analysis (DTMA), the glass transition temperature $T_{g}$ is found to be $-115^{\circ} \mathrm{C}$.

Test samples, also known as poker-chip (Gent \& Lindley 1959), consist of a thin rubber disc bonded between two alloy (Alumec79 ASTM 7022) cylinders. A macroscopic confinement $c$ is defined by means of the disc height $h_{d}$ and the disc radius $r_{d}$ :

$c=\frac{r_{d}}{h_{d}}$

\section{CONFINED COMPRESSION EFFECT}

\subsection{Numerical study}

Above a critical confinement $c^{*}$, stress is essentially hydrostatic in samples under uniaxial loading 


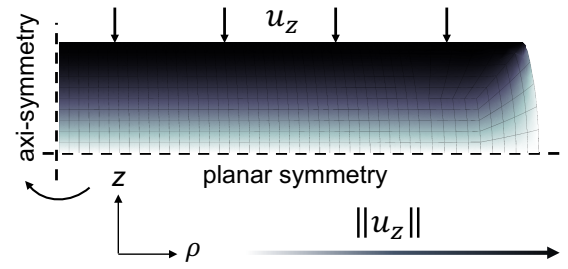

Figure 1: Symmetry and boundary conditions.

(Lindsey 1967). This critical value was investigated through a parametric study conducted in an in-house Finite Element Analysis code (Z-set).

The numerical problem was modelled in an axisymmetric way [Fig. 1]: uniaxial compression simulations were performed by controlling the vertical displacement $u_{z}$. The material was modelled by a quasi-incompressible neo-Hookean formalism where the strain energy potential $W\left(C_{10}, K_{v}\right)$ depends respectively on an elastic and a bulk modulus:

$\rho_{0} W\left(C_{10}, K_{v}\right)=C_{10}\left(I_{1}-3\right)+K_{v} U(J)$

where $C_{10} \sim 1 \mathrm{MPa}, K_{v} \sim 1 \mathrm{GPa}, I_{1}$ is the first strain invariant and $U(J)=\frac{1}{2}\left[\frac{1}{2}\left(J^{2}-1\right)-\log (J)\right]$ takes into account the incompressibility effect. Besides, to avoid volumetric locking and pressure oscillations, a three-field mixed finite element formulation is used (Al Akhrass \& al. 2014).

Simulations were performed on ten numerical samples for a wide range of macroscopic confinements, from 1 to 10 . To analyse the state of stress, the triaxiality ratio (Lemaitre \& Chaboche 1988), defined by the hydrostatic pressure $p_{H}$ and the Von Mises equivalent stress $\sigma_{e q}^{V M}$, was computed:

$\chi=\frac{p_{H}}{\sigma_{e q}^{V M}}$

with $p_{H}=-\frac{1}{3} \operatorname{Tr}(\boldsymbol{\sigma})$ and $\sigma_{e q}^{V M}=\left(\frac{3}{2} \sigma^{d e v}: \sigma^{d e v}\right)^{\frac{1}{2}}$. The following approximation is made. If the specified criterion $\chi>1$ is fulfilled, then the stress tensor is assumed to be hydrostatic.

\subsection{Numerical results}

To compare all results, the volumetric fraction of hydrostatic state $V_{\chi>1}$, i.e. the region of the numerical sample verifying the criterion, is reported [Fig. 2]. The volumetric fraction increases towards 1 . A critical macroscopic confinement $c^{*}=4$ seems to be a relevant threshold, implying at least $80 \%$ of hydrostatic state in the material. Furthermore, a parabolic pressure field along the radial direction $\rho$ was seen. The stress state is not hydrostatic on the free edges because the material is not constrained in the radial direction (Dorfmann \& al. 2002). Therefore, on a sufficiently confined sample, the total stress has a contribution of more than $80 \%$ originating from a hydrostatic state, i.e. a compression load is equivalent to a hydrostatic pressure test.

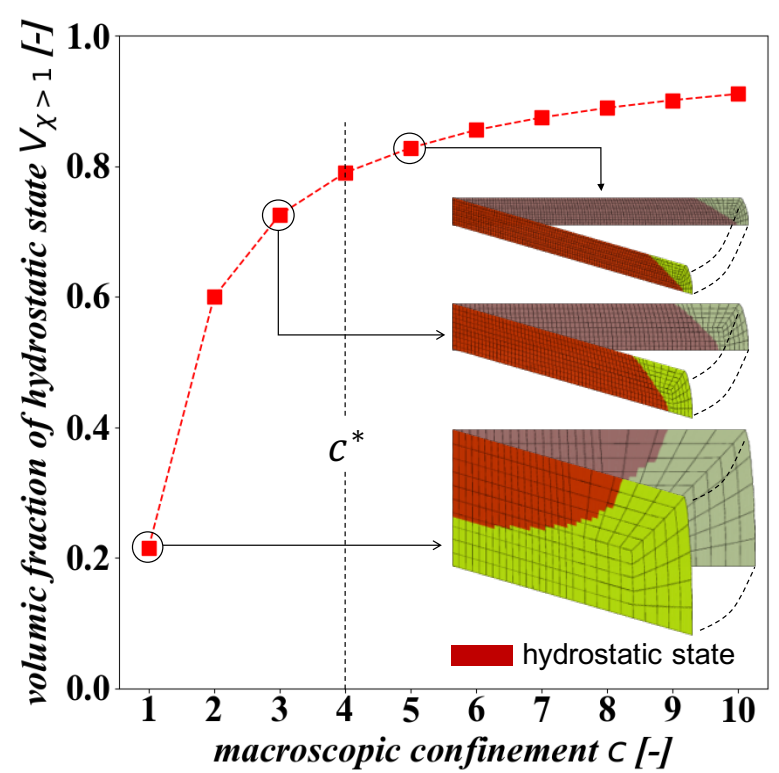

Figure 2: Macroscopic confinement effect on the stress state.

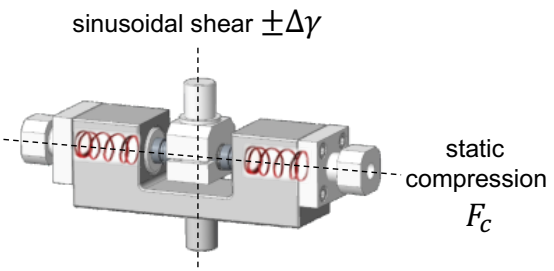

Figure 3: Experimental setup used for shear-compression tests.

\section{PRESSURE EFFECT ON THE THERMO-MECHANICAL BEHAVIOUR}

\subsection{Experimental setup}

A macroscopic confinement of $c=5$ was chosen, such that stress is essentially hydrostatic in the test sample. Shear tests were carried out at controlled temperature $T$ ( -60 to $30^{\circ} \mathrm{C}$ ) and various static compression loads $F_{c}(0$ to $1200 \mathrm{~N})$.

The experimental setup [Fig. 3] was designed to be adapted in an Instron 8801 testing machine $( \pm 50 \mathrm{kN}$, $\pm 75 \mathrm{~mm}$ ). For symmetry reasons, two samples are loaded simultaneously. The hydraulic cylinder displacement, in the perpendicular direction, provides a sinusoidal shear strain amplitude $\Delta \gamma$. To limit heat build-up due to the thermo-mechanical coupling (Ovalle Rodas \& al. 2014), frequency was kept to a minimal value of $1 \mathrm{~Hz}$. The axial spring ensures the static compression load, leading to an average hydrostatic pressure:

$p_{H}=\frac{F_{c}}{\pi r_{d}^{2}}$

Thus, the pressure effect on the thermo-mechanical behaviour can be studied.

\subsection{Experimental results}

Shear hysteresis loops obtained for different applied hydrostatic pressures are highlighted [Fig. 4a.]. Test 
were performed at the same temperature and shear amplitude $\left(T=-15{ }^{\circ} \mathrm{C}, \Delta \gamma=16 \%\right)$. It can be seen that hydrostatic pressure has an increasing effect, even at low values $\sim 1 M P a$, on the thermo-mechanical behaviour of the material: stiffness and dissipation.

The temperature effect on the shear mechanical properties, at a constant hydrostatic pressure, is also investigated. Temperature is seen as an influential parameter with the ability to soften stiffness and to decrease dissipation (Lion 1997).

From these results, both physical parameters, i.e. hydrostatic pressure and temperature, are found to have the same effect on the mechanical behaviour, i.e. two different sets of parameters $\left(p_{H}^{1}, T^{1}\right),\left(p_{H}^{2}, T^{2}\right)$ could be found to exhibit an identical mechanical behaviour [Fig. 4b.]. The existence of a hydrostatic pressure-temperature equivalence is highlighted. According to the complete set of results, the order of magnitude of the hydrostatic pressure effect is about $10 \mathrm{~K}_{\mathrm{MPa}}{ }^{-1}$. Besides, in an analogous study on an pure polyurea (Alkhader \& al. 2012), the hydrostatic pressure effect was found to be much smaller, around $0.24 \mathrm{~K}_{\mathrm{MPa}}^{-1}$, which is consistent with the aforementioned matrix pressure effect existing in pure rubbers. Thus, the present study highlights the fillers major role in the hydrostatic pressure effect.

\section{MICRO-MECHANICAL APPROACH}

\subsection{Physico-mechanical model for filled rubbers}

The next step is to understand, under a physical framework, the hydrostatic pressure effect which was experimentally brought to light, in order to integrate its contribution into a micro-mechanical model, described below.

The addition of fillers into a rubber matrix brings outstanding enhancement in the mechanical properties. This phenomenon is explained by the filler network in which some polymer regions are confined between neighbouring aggregates [Fig. 5]: far away from the filler, the local behaviour is rubbery, similar to pure matrix; by contrast, in confined polymer regions, a strong filler-matrix interaction constricting the polymer to a glassy-like state, i.e. polymer chain mobility reduction, has been demonstrated (Berriot \& al. 2002, Papon \& al. 2012). As the glass transition is related with an increase of mobility, confined polymer regions, also called "glassy bridges", are characterized by a local glass transition temperature $T_{g}{ }^{l o c a l}$. The glassy bridges play a major role in the local behaviour and lead to high dynamics heterogeneities in the filled rubber (Montes \& al. 2003), represented by a glass transition temperature distribution.

A local continuum constitutive equation was formulated. Two main features characterize this model. Firstly, the polymer chain mobility reduction due to the filler-matrix interaction is modelled by a glass transition temperature gradient [Fig. 5]. $T_{g}^{\text {local }}(h)$ re-

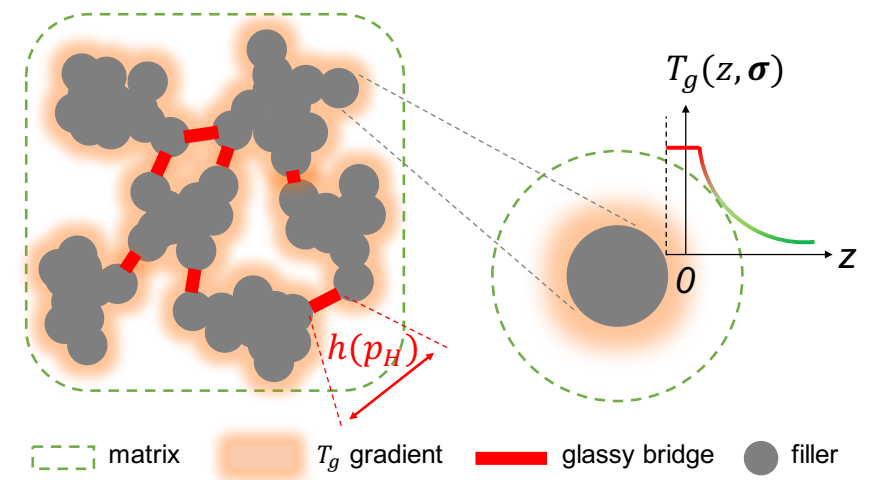

Figure 5: Schematic view of dynamics heterogeneities in filled rubbers.

produces the local glass transition temperature, within the neighbouring aggregates, according to the bridge height $h$ (Long \& Lequeux 2001):

$T_{g}^{\text {local }}(h)=T_{g}^{\text {bulk }}\left(1+\frac{4 \delta}{h}\right)$

where $T_{g}$ bulk is the pure matrix glass transition temperature and $\delta$ is a characteristic length of fillermatrix interaction. Locally, the glass transition takes place at temperatures much higher than in the pure matrix, which reflects the confinement effect.

Secondly, deformation induces an enhancement of mobility of the amorphous macromolecules (Loo \& al. 2000). Thus, the stress affects the chain conformations, modifying the polymer structure from glassy to rubbery (Robertson 1966). The pressure dependence commonly follows the aforementioned matrix pressure effect deduced from the free-volume theory:

$$
\frac{\partial T_{g}}{\partial p}=\frac{\alpha}{K}
$$

Besides, shear stress is proven to affect the glassy polymer dynamic (Lee \& Ediger 2010) but no universal formulation for the deviatoric component has been proposed yet. In polymers, the yield stress is known to obey the pressure modified Von Mises expression (Rottler \& Robbins 2001) normalized by the material parameter $K \sim 1 \mathrm{MPa} . K^{-1}$ (Ward 1983). Yielding is related to an increase of the molecular motion, as well as a decrease of pressure. Thus, in order to take into account volumetric and deviatoric stress, (6) is extended to a general stress expression described with respect to an unstretched state:

$$
T_{g}^{\text {local }}(\boldsymbol{\sigma})=T_{g}^{0}-\frac{\sigma_{e q}^{V M}-\alpha p}{K}
$$

where $T_{g}^{0}$ is the glass transition temperature limit without solicitation. $T_{g}^{\text {local }}(\boldsymbol{\sigma})$ allows to reproduce a glassy-to-rubbery flow-like transition, i.e. a stress softening.

These two contributions on the local glass transition temperature, i.e. the bridge height (5) and the local stress (7), are considered as additive (Colombo \& 

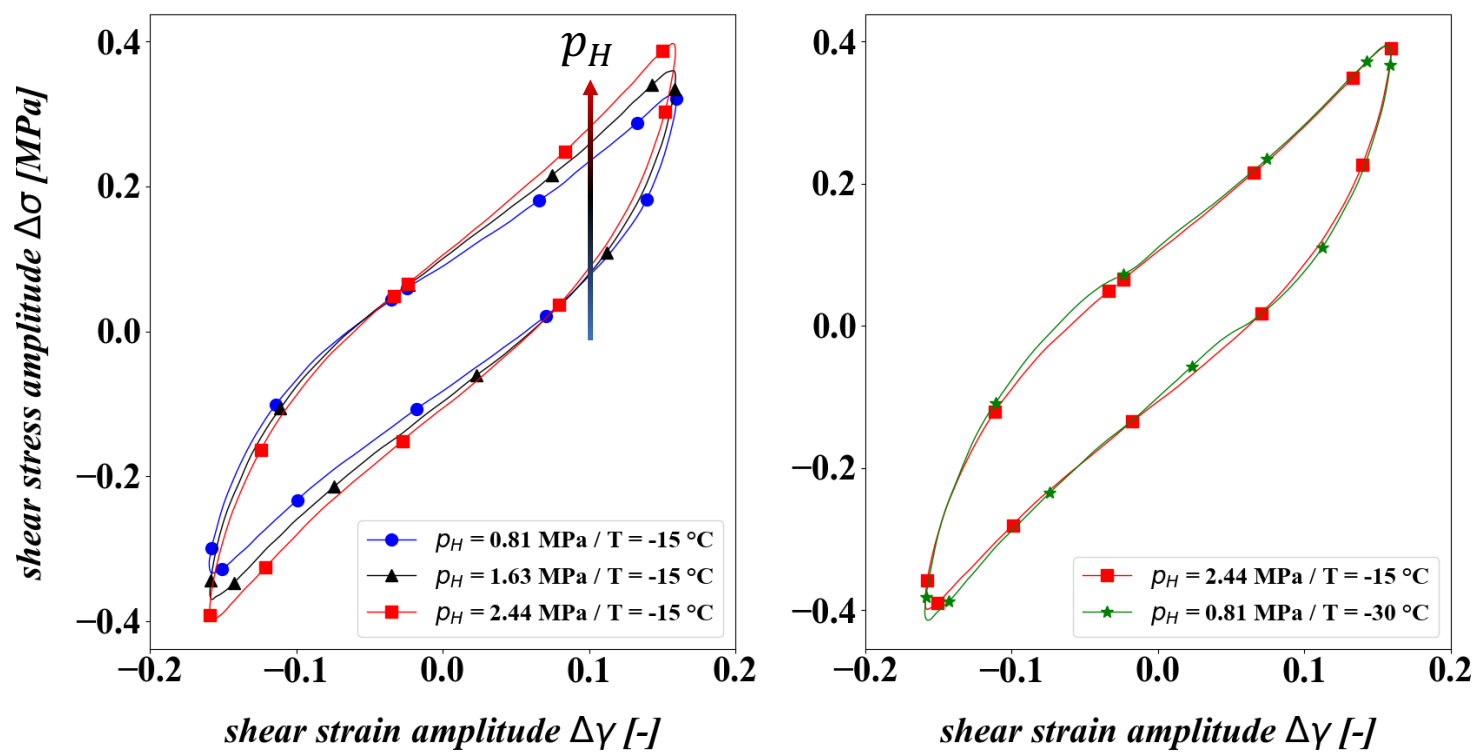

Figure 4: Shear thermo-mechanical response: a) Pressure influence and b) mechanical superposition obtained for two sets $\left(p_{H}, T\right)$.

al. 2015). Thus, a unique glass transition temperature function reflecting the local behaviour is written:

$T_{g}^{\text {local }}(h, \boldsymbol{\sigma})=\underbrace{T_{g}^{\text {bulk }}\left(1+\frac{4 \delta}{h}\right)}_{\text {confinement }}-\underbrace{\frac{\sigma_{e q}^{V M}(h)-\alpha p(h)}{K}}_{\text {stress softening }}$

\subsection{Pressure effect on local behaviour}

For the following, it will be assumed that filled rubber reached the percolation threshold. In this case, the hydrostatic pressure is carried out by the filler network [Fig. 6]. Thus, the bridge height, called $h\left(p_{H}\right)$ in the following, decreases with the hydrostatic pressure.

In order to take into account the hydrostatic pressure dependency, (8) is rewritten as:

$$
\begin{aligned}
T_{g}{ }^{\text {local }}\left(h\left(p_{H}\right), \boldsymbol{\sigma}\right) & =\underbrace{T_{g}^{\text {bulk }\left(1+\frac{4 \delta}{h\left(p_{H}\right)}\right)}}_{T_{g}{ }^{a}} \\
& -\underbrace{\frac{\sigma_{e q}^{V M}\left(h\left(p_{H}\right)\right)-\alpha p\left(h\left(p_{H}\right)\right)}{K}}_{T_{g}{ }^{b}}+\underbrace{\frac{\alpha}{K} p_{H}}_{T_{g}{ }^{c}}
\end{aligned}
$$

Total hydrostatic pressure effect is thus given by three contributions. Their derivative versus hydrostatic pressure gives the amplitude of the hydrostatic pressure effect on the local glass transition temperature for each mechanism:

$\frac{\partial T_{g}{ }^{\text {local }}}{\partial p_{H}}=\frac{\partial T_{g}{ }^{a}}{\partial p_{H}}-\frac{\partial T_{g}{ }^{b}}{\partial p_{H}}+\frac{\partial T_{g}{ }^{c}}{\partial p_{H}}$

where $\frac{\partial T_{g}{ }^{a}}{\partial p_{H}}$ is the contribution on local confinement caused by the bridge height decreasing due to the neighbouring aggregates closer approach, $\frac{\partial T_{g}{ }^{b}}{\partial p_{H}}$ is the contribution on the local stress caused by a stress disruption in the glassy bridge due to high confinement and $\frac{\partial T_{g}{ }^{c}}{\partial p_{H}}$ is the aforementioned matrix pressure effect.
The first two contributions are described in the following subsections. The major contribution will be identified.

\subsubsection{Contribution on local confinement}

A theoretical treatment allows to predict the pressure effect on local confinement. First, the bridge height is assumed to depend on the initial bridge height $h_{0}$ and the local strain:

$h\left(p_{H}\right)=h_{0}\left(1-\varepsilon_{z z}^{\text {local }}\right)=h_{0}\left(1-\frac{\sigma_{z z}^{\text {local }}}{K_{v}}\right)$

where $\varepsilon_{z z}^{\text {local }}$ and $\sigma_{z z}^{\text {local }}$ are respectively the local strain and stress in the bridge direction.

When the hydrostatic pressure acts on the filler network, the force transmitted through the aggregate $F_{z}$ can be expressed at the aggregate scale according to the percolation hypothesis:

$F_{z} \simeq \pi R_{a g}^{2} p_{H}$

and at the bridge scale:

$F_{z} \simeq \pi R_{c}^{2} \sigma_{z z}^{\text {local }}$ with $R_{c}^{2} \simeq R_{p} h_{0}$

where $R_{a g}, R_{p}, R_{c}$ are respectively the aggregate radius, the filler radius and the size of the aforementioned $T_{g}$ gradient.

Combining $\left(9-T_{g}{ }^{a}, 11,12\right)$ and (13) yields:

$T_{g}{ }^{a}=T_{g}{ }^{b u l k}\left(1+\frac{4 \delta}{h_{0}} \cdot \frac{1}{1-\frac{R_{a g}^{2}}{K_{v} R_{p} h_{0}} p_{H}}\right)$

Assuming that $\frac{R_{a g}^{2}}{K_{v} R_{p} h_{0}} p_{H} \ll 1$, one obtains:

$\frac{\partial T_{g}{ }^{a}}{\partial p_{H}} \simeq \frac{4 T_{g}{ }^{\text {bulk }} \delta R_{a g}^{2}}{K_{v} R_{p} h_{0}^{2}}$

which is analytically expressed as a decreasing function of the square of the initial bridge height [Fig. 9]. 


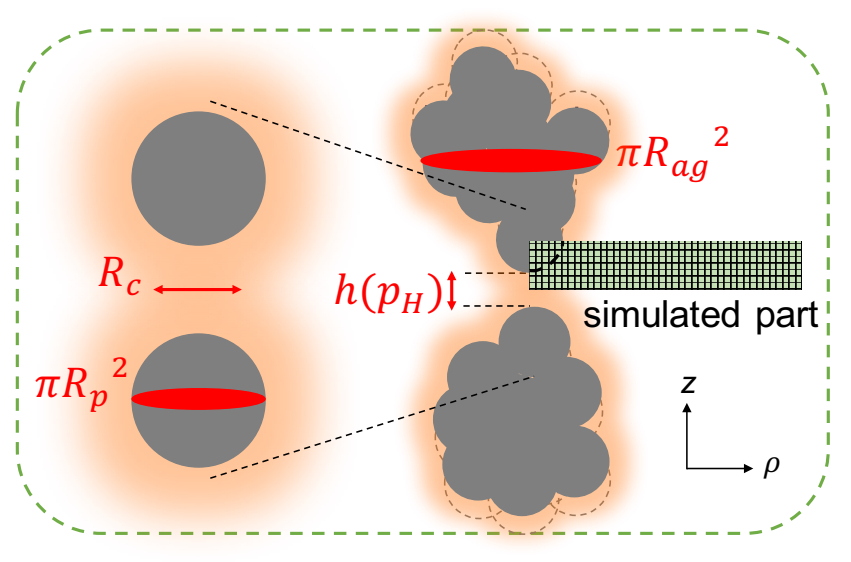

Figure 6: Geometrical parameters and simulated part.

\subsubsection{Contribution on local stress}

On the other side, the pressure effect on local stress is quantified by means of a Z-set analysis. This contribution is approximated to the rate of change of $\left(9-T_{g}^{b}\right)$ with respect to the non-pressurized state:

$$
\frac{\partial T_{g}{ }^{b}}{\partial p_{H}} \simeq \frac{\sigma_{e q}^{V M}\left(h\left(p_{H}\right)\right)-\alpha p\left(h\left(p_{H}\right)\right)}{K p_{H}}
$$

It is computed in the confined region through a parametric study on the initial bridge height. It consists in simulating the closer approach of two aggregates due to hydrostatic pressure [Fig. 6] to quantify the stress disruption effect in the confined region. The numerical model is axi-symmetric: uniaxial compression simulations were performed by controlling the hydrostatic pressure acting on the aggregates. In a similar way as the initial numerical study, the matrix is modelled by a neo-Hookean formalism and the elastic modulus $C_{10}$ dependence is studied. Besides, the filler is assumed to be a stiffer elastic material $(E=70 G P a, v=0.17)$.

For an infinite bridge height $h_{0} \rightarrow \infty$, i.e. no confinement, a homogeneous uniaxial compression state is expected everywhere in the matrix:

$\boldsymbol{\sigma}=\left[\begin{array}{ccc}0 & 0 & 0 \\ 0 & 0 & 0 \\ 0 & 0 & -\sigma_{z z}\end{array}\right] \Rightarrow\left\{\begin{array}{l}p=\frac{\sigma_{z z}}{3} \\ \sigma_{e q}^{V M}=\sigma_{z z}\end{array}\right.$

In this limit case, the asymptotic behaviour is thus found to be: $\frac{\partial T_{g}{ }^{b}}{\partial p_{H}} \rightarrow 2.7 \mathrm{~K}_{\mathrm{MPa}} \mathrm{MP}^{-1}$ [Fig. 9].

For bridges of interest, i.e. 0.75 to $8 \mathrm{~nm}$, the triaxiality ratio [Fig. 7] never exceeds $\frac{1}{\alpha}$ in confined regions, i.e. $\rho<R_{p}$, further:

$\chi<\frac{1}{\alpha} \Rightarrow \sigma_{e q}^{V M}-\alpha p>0$

which induces a negative contribution of the local stress on the total hydrostatic pressure effect (10). Furthermore, this quantity is found to be strictly independent of the elastic modulus, i.e. rubbery-like or glassy-like.

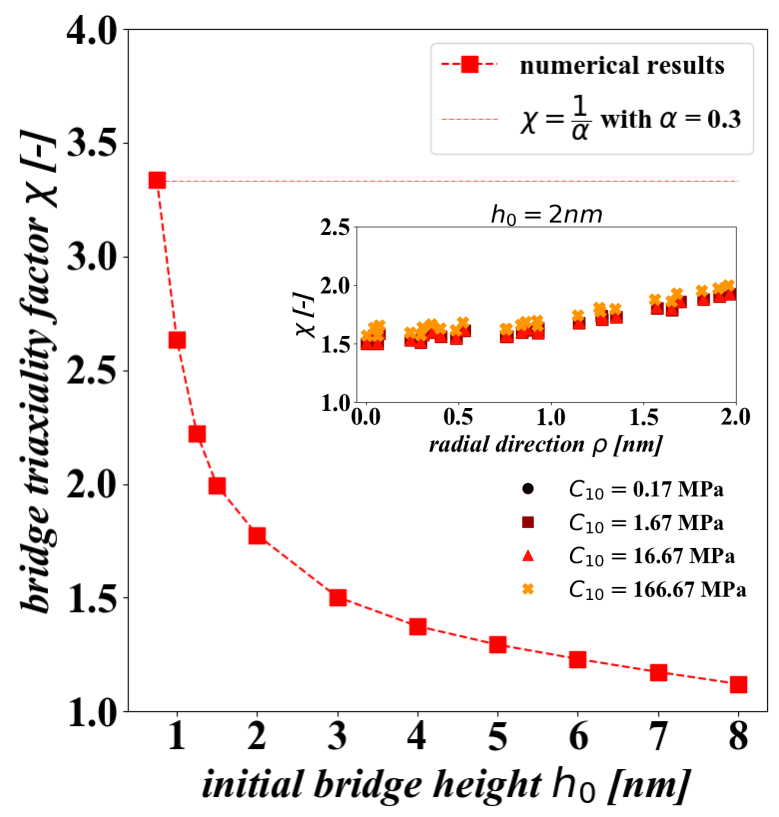

Figure 7: Triaxiality ratio in the confined regions (bridges).

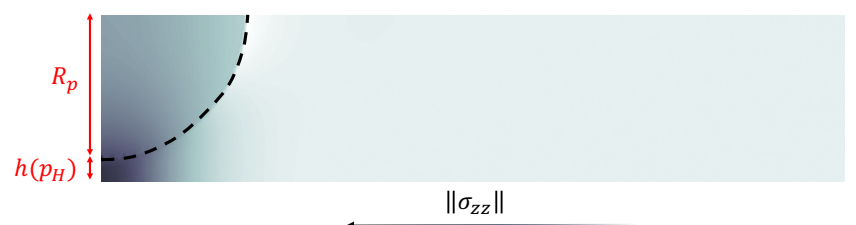

Figure 8: Unixial stress field $\sigma_{z z}$ for $h_{0}=2 \mathrm{~nm}, R_{p}=5 \mathrm{~nm}$.

The uniaxial stress field [Fig. 8] obtained for an initial glassy bridge $h_{0}=2 \mathrm{~nm}$ allows to clearly emphasize the local stress disruption in the confined region which is responsible for this pressure effect. By contrast, a homogeneous uniaxial compression state is observed if sufficiently far away from the bridge area, i.e. $\rho>R_{p}$. Numerical results [Fig. 9] show a sharp increase of the pressure effect on the local stress for very tiny bridges $\left(h_{0}<1.5 \mathrm{~nm}\right)$. For higher bridges, a slight decrease, consistent with the aforementioned asymptotic behaviour (17), is observed.

\subsubsection{Discussion}

From a micro-mechanics framework, hydrostatic pressure brings a contribution on local confinement, on local stress and on the matrix. All these contributions were quantified [Fig. 9]. It clearly appears that the local confinement effect, due to the closer approach of aggregates, dominates.

The total hydrostatic pressure effect (10) accounts for the local glass transition temperature shift expected for filled rubbers [Fig. 9]. A local shift of about $10 \mathrm{~K}_{\mathrm{MPa}} \mathrm{MP}^{-1}$ is predicted for intermediate bridge heights $3 \mathrm{~nm} \leq h \leq 4 \mathrm{~nm}$, which is consistent with our experimental observations. This hydrostatic pressure effect is about two orders of magnitude larger than the one measured in pure rubbers.

From a physical framework, the hydrostatic pressure influences the initial filler network by decreasing the bridge heights (closer approach of aggregates). It leads to a significant shift of the local be- 


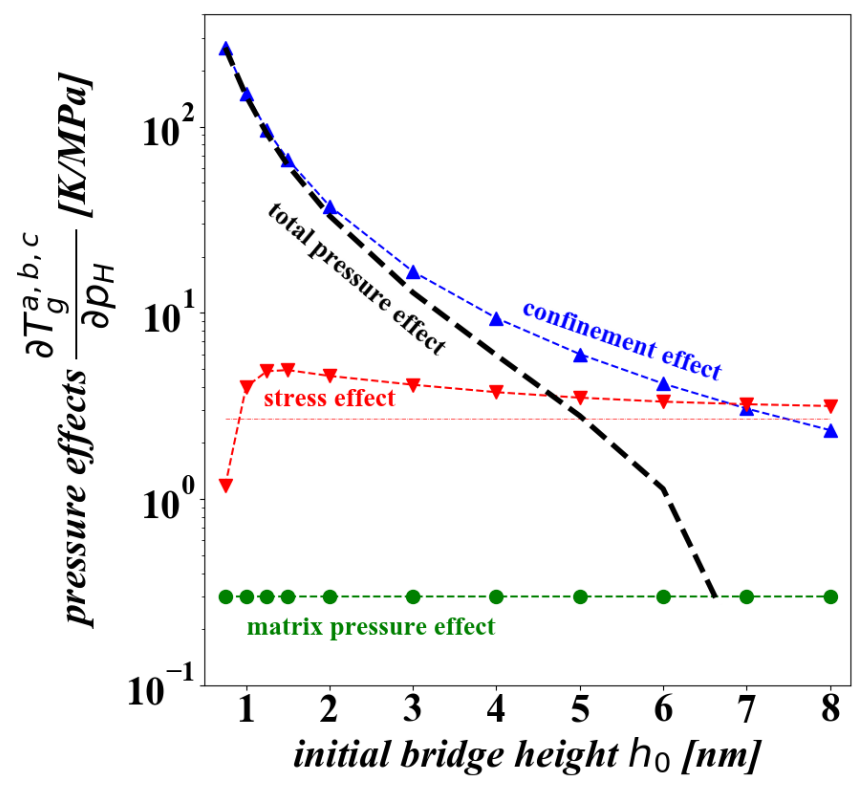

Figure 9: Total hydrostatic pressure effect on the local glass transition temperature with respect to the initial bridge height.

haviour $T_{g}^{\text {local }}$ for shorter bridges. This mechanism drives the hydrostatic pressure-temperature superposition observed on the real sample. Finally, in order to describe quantitatively the macroscopic behaviour of the material, a scale-up has to be done, which is in progress.

\section{CONCLUSIONS}

In order to study the hydrostatic pressure effect on the thermo-mechanical behaviour of filled rubbers, an experimental setup was designed. Shear mechanical properties have been measured under superposed hydrostatic pressure and temperature. A hydrostatic pressure-temperature superposition is highlighted. The amplitude of this effect is about $10 \mathrm{~K} \cdot \mathrm{MPa}^{-1}$, which is two orders of magnitude larger than the one observed in pure rubbers.

From a micro-mechanics framework, the local glass transition temperature shift involved by the hydrostatic pressure -through local confinement, local stress and the matrix- has been discussed. Our results proved that the local confinement effect, due to the closer approach of aggregates, is essentially responsible for this local shift. More importantly, this predicted shift is consistent with our experimental results, which explains the observed hydrostatic pressure-temperature superposition.

\section{REFERENCES}

Al Akhrass, D. \& al. (2014). Integrating a logarithmic-strain based hyperelastic formulation into a three-field mixed finite element formulation to deal with incompressibility in finitestrain elastoplasticity. Finite Elements in Analysis and Design 86, 61-70.

Alkhader, M. \& al. (2012). A new shear-compression test for determining the pressure influence on the shear response of elastomers. Experimental Mechanics 52(8), 1151-1161.
Berriot, J. \& al. (2002). Filler-elastomer interaction in model filled rubbers, a $1 \mathrm{~h} \mathrm{nmr}$ study. Journal of Non-Crystalline Solids 307, 719-724.

Bills, K. W. \& al. (1960). The tensile properties of highly filled polymers. effect of filler concentrations. Journal of Applied Polymer Science 4(12), 259-268.

Cheng, Z.-Y. \& al. (1999). Pressure-temperature study of dielectric relaxation of a polyurethane elastomer. Journal of Polymer Science 37(10), 983-990.

Colombo, D. \& al. (2015). Towards constitutive equation for non-linear mechanics of pdms-nanosilica. European Conference on Constitutive Models for Rubbers.

Dorfmann, A. \& al. (2002, Apr). Shear, compressive and dilatational response of rubberlike solids subject to cavitation damage. International Journal of Solids and Structures 39(7), 1845-1861.

Ferry, J. D. \& R. A. Stratton (1960). The free volume interpretation of the dependence of viscosities and viscoelastic relaxation times on concentration, pressure, and tensile strain. Kolloid-Zeitschrift 171(2), 107-111.

Fillers, R. W. \& N. W. Tschoegl (1977). The effect of pressure on the mechanical properties of polymers. Transactions of the Society of Rheology 21(1), 51-100.

Gent, A. N. \& P. B. Lindley (1959). Internal rupture of bonded rubber cylinders in tension. The Royal Society 249(1257), 195-205.

Lee, H.-N. \& M. D. Ediger (2010). Interaction between physical aging, deformation, and segmental mobility in poly (methyl methacrylate) glasses. The Journal of chemical physics 133(1), 014901.

Lemaitre, J. \& J.-L. Chaboche (1988). Mécanique des matériaux solides. Dunod.

Lindsey, G. H. (1967). Triaxial fracture studies. Journal of Applied Physics 38(12), 4843-4852.

Lion, A. (1997). On the large deformation behaviour of reinforced rubber at different temperatures. Journal of the Mechanics and Physics of Solids 45(11-12), 1805-1834.

Long, D. \& F. Lequeux (2001). Heterogeneous dynamics at the glass transition in van der waals liquids, in the bulk and in thin films. The European Physical Journal E 4(3), 371-387.

Loo, L. S. \& al. (2000). Chain mobility in the amorphous region of nylon 6 observed under active uniaxial deformation. Science 288(5463), 116-119.

Montes, H. \& al. (2003). Influence of the glass transition temperature gradient on the nonlinear viscoelastic behavior in reinforced elastomers. Macromolecules.

Ovalle Rodas, C. \& al. (2014). A finite strain thermo-viscoelastic constitutive model to describe the self-heating in elastomeric materials during low-cycle fatigue. Journal of the Mechanics and Physics of Solids 64, 396-410.

Papon, A. \& al. (2012). Glass-transition temperature gradient in nanocomposites: evidence from nuclear magnetic resonance and differential scanning calorimetry. Physical review letters 108(6), 065702.

Paterson, M. S. (1964). Effect of pressure on young's modulus and the glass transition in rubbers. Journal of Applied Physics 35(1), 176-179.

Robertson, R. E. (1966). Theory for the plasticity of glassy polymers. The Journal of Chemical Physics 44(10), 3950-3956.

Rottler, J. \& M. O. Robbins (2001). Yield conditions for deformation of amorphous polymer glasses. Physical Review E 64(5), 051801.

Ward, I. (1983). Mechanical properties of solid polymers, 2nd ed. John Wiley and Sons. 